\title{
Method for Classification \\ of Objects Optoelectronic Exploration Systems \\ Based Multispectral Cuboid Images
}

\author{
Igor N. Ischuk ${ }^{\mathrm{a}, \mathrm{b}}$, \\ Evgeniy A. Stepanov a, Andrey A. Bebenina, \\ Dmitry D. Dmitriev ${ }^{b}$ and Andrey M. Filimonov ${ }^{a}$ \\ ${ }^{a}$ Military Education and Research Centre of Military-Air Forces \\ «Military-Air Academy \\ Named After Professor N.E. Zhukovsky and Yu.A. Gagarin» \\ 54a Starykh Bolshevikov Str., Voronezh, 394064, Russia \\ ${ }^{b}$ Siberian Federal University \\ 79 Svobodny, Krasnoyarsk, 660041, Russia
}

Received 04.12.2016, received in revised form 10.01.2017, accepted 28.02.2017

The article deals with the classification of objects opto-electronic intelligence systems on the basis of processing of multispectral images cuboid, technology generation and processing cuboid multispectral images of man-made objects in the course of daily observations. A general statement of the problem according to the classification of dynamic images in the infrared and visible wavelength ranges. The results of the implementation of the method of classification in the field experiment.

Keywords: multispectral imaging, thermal tomography, thermal radiation, thermal contrast, dynamic IR signature, thermal conductivity material, cuboid IR image, hidden objects.

Citation: Ischuk I.N., Stepanov E.A., Bebenin A.A., Dmitriev D.D. Filimonov A.M. Method for classification of objects optoelectronic exploration systems based multispectral cuboid images, J. Sib. Fed. Univ. Eng. technol., 2017, 10(2), $183-190$. DOI: 10.17516/1999-494X-2017-10-2-183-190.

(c) Siberian Federal University. All rights reserved

* Corresponding author E-mail address: boerby@rambler.ru, Nikk_444@mail.ru 


\title{
Способ классификации объектов
}

оптико-электронными системами разведки

на основе обработки

\section{многоспектрального кубоида изображений}

\author{
И.Н. Ищук $\kappa^{\mathrm{a}, \boldsymbol{0}}$, Е.А. Степанов ${ }^{\mathrm{a}}$, \\ А.А. Бебенин ${ }^{\sigma}$, Д.Д. Дмитриев ${ }^{\mathrm{a}}$, А.М. Филимонов ${ }^{\mathrm{a}}$ \\ ${ }^{a}$ Военный учебно-научный иеентр Военно-воздушных сил \\ «Военно-воздушная академия \\ имени профессора Н.Е. Жуковского и Ю.А. Гагарина» \\ Россия, 394064, Воронеж, ул. Старых Большевиков, 54 а \\ ${ }^{6}$ Сибирский федеральный университет \\ Россия, 660041, Красноярск, пр. Свободньій, 79
}

В статье рассматривается классификация объектов оптико-электронными системами разведки на основе обработки многоспектрального кубоида изображений, технология формирования и обработки кубоида многоспектральных изображений техногенных объектов в ходе суточных наблюдений. Представлена общая постановка задачи классификации по данным динамических изображений в инфракрасном и видимом диапазонах длин волн. Приведены результать реализации предложенного способа классификации в ходе натурного эксперимента.

Ключевые слова: многоспектральные изображения, тепловая томограмма, тепловое излучение, тепловой контраст, динамические ИК-сигнатуры, теплопроводность материалов, кубоид ИК-изображения, скрытые объекты.

\section{Введение}

Современные оптико-электронные системы (ОЭС) разведки наземных техногенных объектов, установленные на беспилотных летательных аппаратах (БЛА) и работающие в различных диапазонах электромагнитного спектра, осуществляют поиск объектов путем анализа возникающих яркостных контрастов. На эти системы воздействуют различные факторы, такие как параметры съемки (высота, скорость и эволюции полета БЛА, освещенность сцены), погодные, антропогенные и другие воздействия. ОЭС инфракрасного (ИК) диапазона длин волн характеризуются способностью обнаружения по тепловым контрастам малозаметных объектов, менее подвержены погодным воздействиям и не зависят от присутствия внешних источников излучения [1]. При этом современные ОЭС как в видимом, так и в ИК-диапазонах характеризуются возможностью получения видеоизображения качества FullHD (разрешением 1920x1080 пиксель) и выше [2].

Применение различных методов обзора поверхности Земли при однократной съемке не всегда позволяет обнаружить малозаметный техногенный объект. Известны различные способы решения задач обнаружения и распознавания малозаметных объектов в ИК-диапазоне длин волн в ходе дистанционного мониторинга Земли с применением БЛА, однако они не используют свойства информационной избыточности динамических изображений. 
Современные БЛА большой продолжительности полета позволяют вести поиск малозаметных техногенных объектов в течение длительного времени с переходом от светлого времени суток к темному, фиксируя изображения местности с определенным интервалом. В результате получается набор динамических изображений, обладающий информационной избыточностью.

Использование операторного метода дешифрирования динамических изображений больших участков местности характеризуется большими психофизическими нагрузками и низкой эффективностью обнаружения малозаметных объектов. Для решения задачи снижения нагрузки на дешифровщика при обнаружении малозаметных объектов следует использовать алгоритмы автоматизации процесса обработки набора динамических многоспектральных изображений (кубоида многоспектральных изображений), содержащего избыточную информацию об изменении поля яркости наблюдаемой местности. В процессе обработки динамических ИК-изображений можно получить тепловую томограмму местности [3], отражающую распределение теплофизических параметров объектов. В видимом диапазоне длин волн достаточно отобрать наиболее контрастное изображение, поскольку малозаметные техногенные объекты в данной области электромагнитного спектра не меняют свои отражательные свойства при условии постоянства погоды.

\section{Постановка задачи}

При периодическом дистанционном измерении тепловых полей тепловизионным приемником получают детерминированные значения радиационных температур $\mathbf{T}_{r}^{1}, \mathbf{T}_{r}^{2}, \ldots, \mathbf{T}_{r}^{k}$, там радиационная температура каждой области поверхности на изображении - пикселе - представляется фиксированным значением $\mathbf{T}_{r}[m, n: k]$, где $m, n$ - пространственные отсчеты, $m \in \overline{0, M}$; $n \in \overline{0, N} ; k \in \overline{1, K}$ - отсчеты по времени.

Математическая задача классификации формулируется с помощью дискриминантной функции. Пусть $\omega_{1}, \omega_{2}, \ldots, \omega_{\zeta}$ обозначают $\zeta$ классов (образов), подлежащих распознаванию и образующих полную группу событий $\left\{\omega_{i}\right\}_{i=1}^{\varsigma}$ таких, что $\forall_{i} P\left(\omega_{i}\right)>0, \forall j \neq i, \omega_{i} \cap \omega_{j} \neq 0, \sum_{i=1}^{\varsigma} \omega_{i}=\Omega[4]$.

Допустим, что кубоид ИК-изображений описывается вектором $\widetilde{\mathbf{T}}=\left[\mathbf{T}_{r}^{1}, \mathbf{T}_{r}^{2}, \ldots, \mathbf{T}_{r}^{k}\right]^{\mathrm{T}}$. Так как $\widetilde{\mathbf{T}}$ является стохастической функцией времени и зависит от большого числа внешних факторов, снижение неопределенности в получении оценки принадлежности образа к классу $\omega_{i}$ может быть достигнуто путем редукции кубоида ИК-изображения и получения изображения пространственного распределения теплопроводности $\lambda[m, n]: \boldsymbol{\lambda}=\mathbf{A}^{-1}(\widetilde{\mathbf{T}})$, где $\mathbf{A}^{-1}$ - обратный оператор, реализующий решение КОЗТ. Оператор $\mathbf{A}^{-1}$ можно реализовать путем численного решения оптимизационной задачи $\hat{\lambda}=\arg \min _{\lambda \in \mathrm{R}}\|\mathbf{T}-\widetilde{\mathbf{T}}\|$, где $\mathbf{T}-$ кубоид термодинамических температур, рассчитанных в соответствии с численным решением ПЗТ методом разностных схем: $\mathbf{T}^{k-1}=\mathbf{A} \cdot \mathbf{T}^{k}$, где A - матрица-определитель разностной схемы. Представим функцию правдоподобия $L(\hat{\lambda} \mid \omega): \Omega \rightarrow \mathrm{R}$, где $\hat{\lambda} \in \mathrm{R}$. Тогда точечная оценка принадлежности пикселя на изображении тепловой томограммы к классу $\omega_{i}$ будет определяться оценкой максимального правдоподобия параметра $\omega$ на основе редукции кубоида ИК-изображений [4]. 
Повышение вероятности обнаружения малозаметных техногенных объектов достигается использованием на БЛА многоспектральных оптико-электронных систем (МОЭС), обеспечивающих совместную обработку полей яркостей в различных диапазонах длин волн. Оценка принадлежности пикселя изображения в МОЭС в ИК- и видимом диапазонах длин волн к классу $\omega_{i}$ реализуется путем минимизации соответствующей целевой функции $U$, основанной на вычислении эвклидовой метрики. На основании теоретических и экспериментальных исследований, представленных в работе [4], рассматриваемая задача классификации объектов в МОЭС будет иметь вид

$$
\left\{\begin{array}{l}
\Omega=\arg \min _{\omega \in \Omega} U\left(\sum_{i=1}^{\varsigma}\left(\sum_{j=1}^{l}\left(\hat{\omega}_{i}^{j}-\omega_{q}^{j}\right)^{2}\right)^{0,5}\right) ; \\
\hat{\omega}=\arg \max _{\omega \in \Omega} L\left(\hat{\lambda}_{1}, \hat{\lambda}_{2}, \ldots, \hat{\lambda}_{\varsigma} \mid \omega\right) ; \\
\hat{\lambda}=\arg \min _{\lambda \in \mathrm{R}}\|\mathbf{T}-\widetilde{\mathbf{T}}\| ; \\
\mathbf{T}^{k-1}=\mathbf{A} \cdot \mathbf{T}^{k}
\end{array}\right.
$$

где $U$ - оператор разбиения множества объектов $\Omega$ на изображениях, полученных в видимом и ИК-диапазонах длин волн в результате обработки кубоида изображений; $l$ - количество плоскостей (поле яркости изображения тепловой томограммы, поле яркости изображения в видимом диапазоне длин волн); $q \in \overline{1, \varsigma}$.

\section{Алгоритм классификации}

Этапы реализации способа классификации объектов МОЭС на основе обработки многоспектрального кубоида изображений в соответствии с (1) представляются в виде набора конечных операций, выполняемых в такой последовательности:

Шаг 1. С установленной на борту БЛА МОЭС с определенной периодичностью в течение суток производится съемка исследуемого участка местности.

Шаг 2. По данным полученных динамических ИК-сигнатур строится кубоид ИКизображений и производится перерасчет значений радиационных температур в значения избыточных температур [5].

Шаг 3. По данным кубоида ИК-изображений с использованием эталонных материалов решается прямая задача теплопроводности (ПЗТ) на основе анизатропного представления приповерхностного слоя Земли.

Шаг 4. С использованием методов многопараметрической оптимизации решается коэффициентная обратная задача теплопроводности (КОЗТ) с составлением и минимизацией функционала невязки по рассчитанным и экспериментально измеренным значениям температур для каждого эталонного материала и получаются оцененные значения параметров, аппроксимирующих функцию источника тепла и коэффициентов зависимости ТФП от температуры $[5,6]$.

Шаг 5. С использованием матрицы приближенности областей изображения к эталонным материалам решается КОЗТ путем минимизации квадратичного функционала невязки по рассчитанным и экспериментально измеренным значениям избыточных температур попиксельно для всего растра изображений, по результатам решения КОЗТ строят пространственное распределение ТФП - тепловую томограмму [5].

$$
-186-
$$


Шаг 6. Из динамического набора изображений видимого диапазона длин волн выделяется наиболее контрастное изображение.

Шаг 7. Производится кластеризация тепловой томограммы и изображения, полученного в видимом диапазоне длин волн [7].

Шаг 8. По данным кластеризованных изображений выделяются зоны подповерхностных неоднородностей, обусловленных несоответствием модели ПЗТ (шаг 3) данным дистанционных измерений.

Шаг 9. В выделенных зонах подповерхностных неоднородностей производится перерасчёт оценённых значений поля яркостей (шаг 3 - шаг 5) с учетом изотропного представления слоя Земли.

\section{Экспериментальная апробация способа классификации}

Для экспериментальной апробации способа классификации МОЭС разведки объектов с учетом фона и эталонных материалов в естественных условиях была рассчитана томограмма на основе набора многоспектральных изображений одного участка местности, полученных в ходе периодической съемки МОЭС разведки с БЛА квадрокоптерного типа (рис. 1). В качестве эталонных материалов были использованы дерево (сосна) $(\lambda=0.18 \mathrm{BT} / \mathrm{M} \cdot \mathrm{K}$, $\mathrm{c}=2300$ Дж/кг $\cdot \mathrm{K}$ - теплопроводность, удельная теплоемкость), сталь ( $\lambda=58 \mathrm{BT} / \mathrm{M} \cdot \mathrm{K}$, $\mathrm{c}=482$ Дж/кг $\cdot \mathrm{K})$, пенопласт $(\lambda=0.04 \mathrm{BT} / \mathrm{M} \cdot \mathrm{K}, \mathrm{c}=1400$ Дж/кг $\cdot \mathrm{K})$, пенобетон $(\lambda=0.18 \mathrm{BT} / \mathrm{M} \cdot \mathrm{K}$, $\mathrm{c}=840$ Дж/кг К $)$ и гранит $(\lambda=3.5 \mathrm{BT} / \mathrm{M} \cdot \mathrm{K}, \mathrm{c}=880$ Дж/кг $\cdot \mathrm{K})$, плита из красного полнотелого кирпича $(\lambda=0.67 \mathrm{BT} / \mathrm{M} \cdot \mathrm{K}, \mathrm{c}=860$ Дж/кг $\cdot К)$. Съемка велась оптическим сенсором Sony

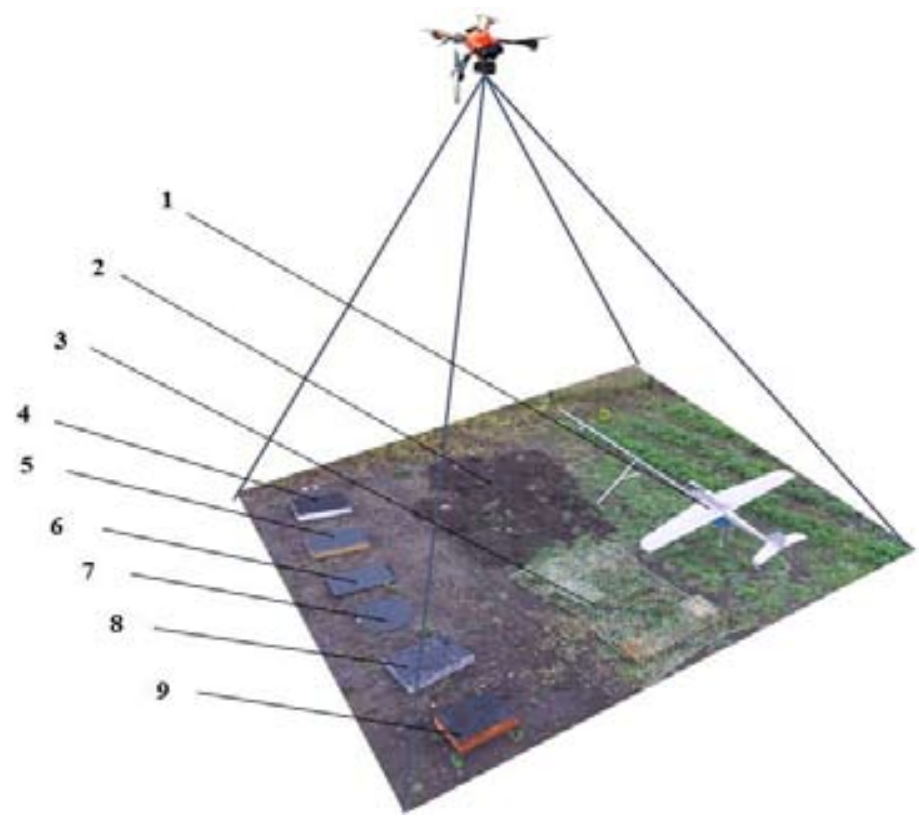

Рис. 1. Модельная обстановка проведения натурного эксперимента: 1 - БЛА на позиции; 2 - минное поле; 3 - объекты, скрытые маскировочной сетью; 4 - пенобетон; 5 - дерево (сосна); 6 - гранит; 7 - сталь; 8 - пенопласт; 9 - красный полнотелый кирпич 

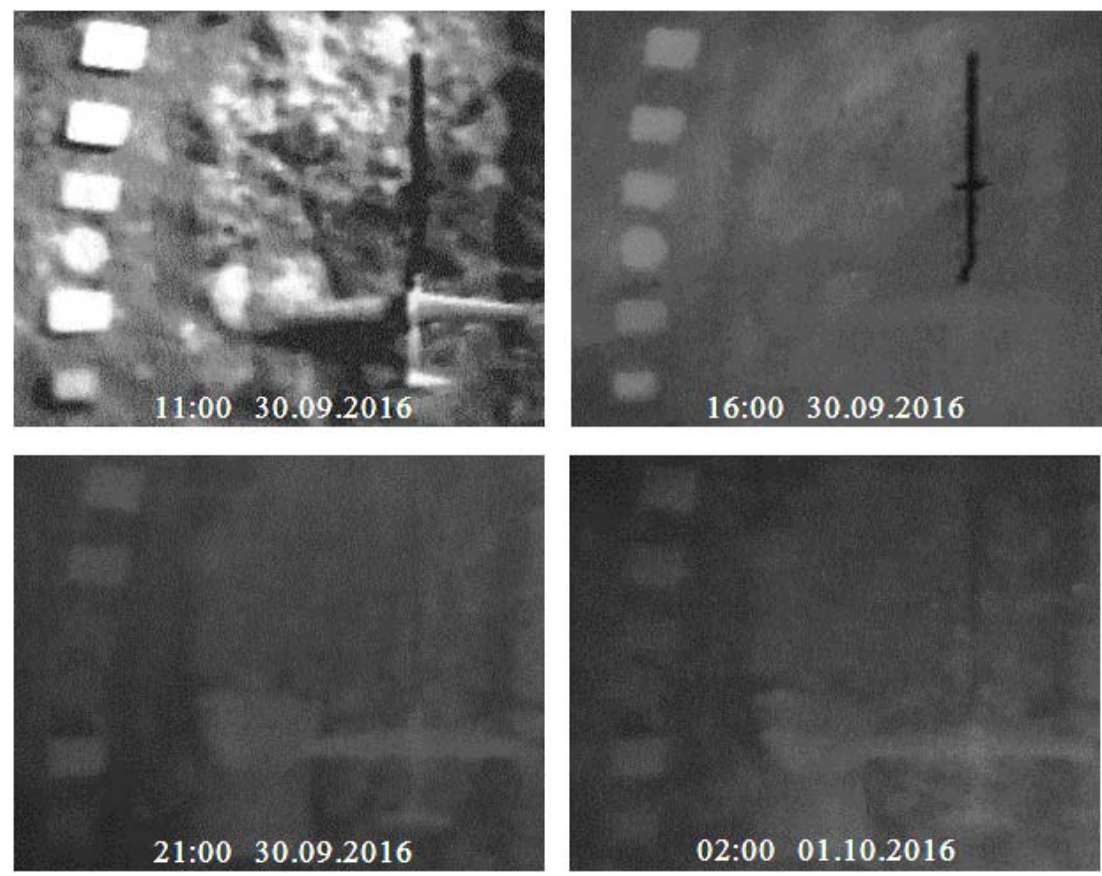

Рис. 2. Ряд ИК-изображений, полученных в течение суток

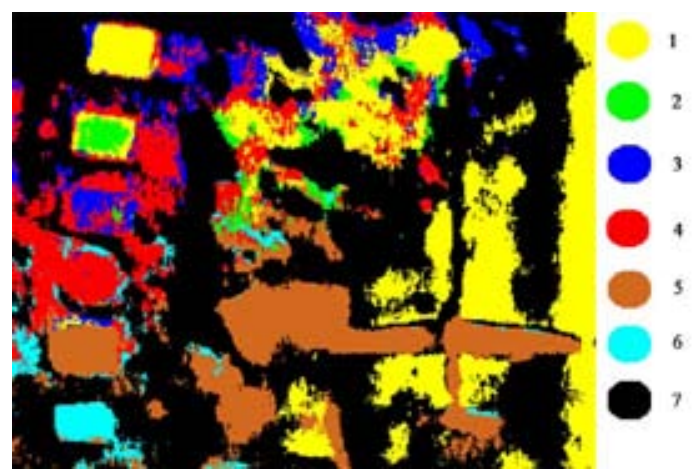

a

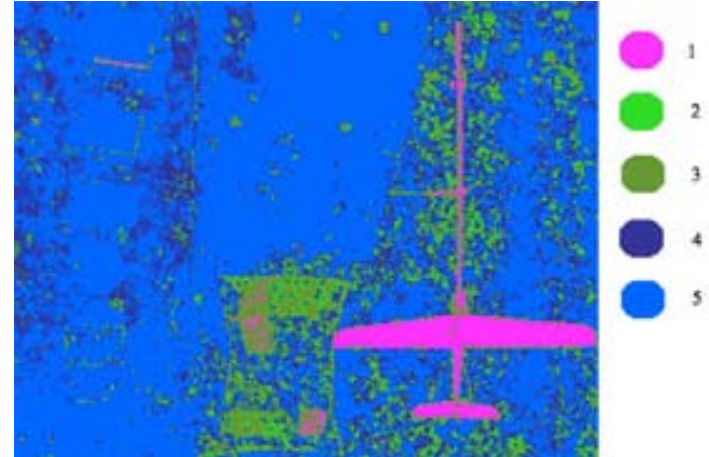

6

Рис. 3. Кластеризованные изображения: а - тепловой томограммы, полученной по данным динамических ИК-изображений для классов материалов: 1 - пенобетон; 2 - дерево; 3 - гранит; 4 - железо; 5 - пенопласт; 6 - красный полнотелый кирпич; 7 - грунт; б - видимого изображения с распределением классов по значению яркости равномерно в пяти диапазонах от 0 до 255

EXMOR 1/2.3" и тепловизионным приемником Flir Tau2 с высоты 20 м в течение суток с интервалом 1 ч. В результате съемки всего было получено 24 ИК-изображения. Ряд из них представлен на рис. 2.

Результатом обработки полученного кубоида ИК-изображений является тепловая томограмма, кластеризованный вид которой представлен на рис. За. Кластеризованное изображение наблюдаемой модельной сцены в видимом диапазоне длин волн дано на рис. 3б. В результате анализа полученных кластеризованных изображений выделе- 


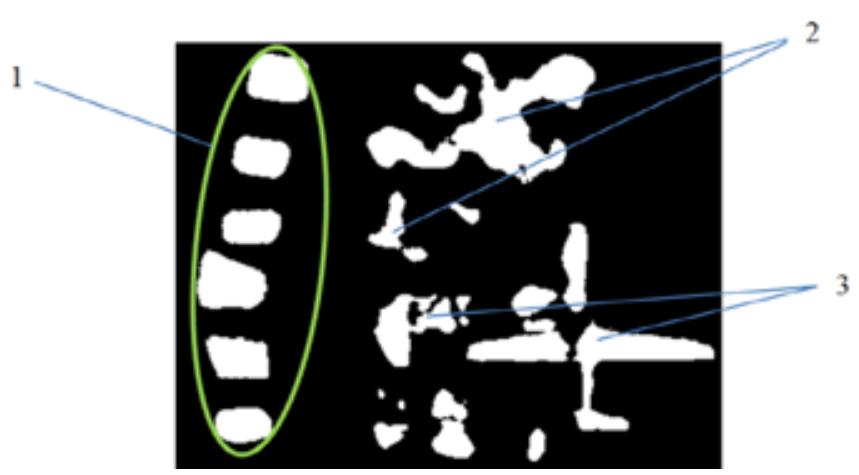

Рис. 4. Зоны изотропных (ортотропных) объектов: 1 - зона многослойной структуры «эталонные объекты на грунте»; 2 - зона анизотропных «подповерхностных объектов»; 3 - зона многослойных ортотропных объектов БЛА

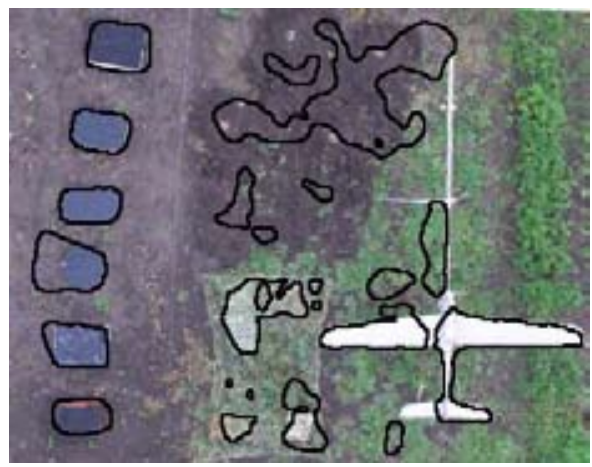

Рис. 5. Совмещенное изображение видимого диапазона длин волн и выделенных зон ортотропных объектов

ны зоны изотропных (ортотропных) объектов [8] (рис. 4). Совмещенное изображение видимого диапазона длин волн и выделенных зон ортотропных объектов приведено на рис. 5.

\section{Вывод}

Использование предложенного способа классификации объектов МОЭС разведки на основе обработки многоспектрального кубоида изображений позволило в ходе натурного эксперимента определить зоны несоответствия математической модели ПЗТ данным дистанционных измерений МОЭС на БЛА. Таким образом, для более эффективного решения задачи классификации объектов по данным динамических изображений с построением тепловых томограмм [4] при решении КОЗТ необходимо применять изотропную модель подповерхностных объектов, находящихся в грунте, с учетом априорных данных о геометрических параметрах объектов, расположенных на поверхности, получаемых путем анализа изображений в видимом диапазоне длин волн.

Работа выполнена при финансовой поддержке РФФИ (грант № 15-08-02611 А). 


\section{Список литературы}

[1] Jiayi Ma, Chen Chen, Chang L, Jun Huang. Infrared and visible image fusion via gradient transfer and total variation minimization. Information Fusion, 2016, 31, 100-109.

[2] Dhaval Shingala, Rashmi Agrawal. Super-Resolution of Multispectral Images. International Journal for Scientific Research \& Development, 2013, 1(3), 770-774.

[3] Ищук И.Н., Обухов В.В., Парфирьев А.В., Филимонов А.М. Методика дистанционного контроля изотропных материалов путем редукции кубоида ИК-изображений. Измерительная техника, 2014, 9, 41-45 [Ischuk I.N., Obukhov V.V., Parfir'ev A.V., Filimonov A.M. Method of remote control of isotropic materials by reduction cuboid infrared images. Measurement technology, 2015, 9, 41-45 (in Russian)]

[4] Ищук И.Н., Филимонов А.М., Степанов Е.А., Постнов К.В. Способ классификации стационарных и квазистационарных объектов по данным динамических инфракрасных изображений, получаемых комплексами с беспилотными летательными аппаратами. Радиотехника, 2016, 10, 145-153 [Ischuk I.N., Filimonov A.M., Stepanov E.A., Postnov K.V. Method of classification of stationary and non-stationary objects according to the dynamic infrared images obtained complexes with unmanned aerial vehicles. Radioengineering, 2016, 10, 145-152 (in Russian)]

[5] Ищук И.Н., Парфирьев А.В. Реконструкция кубоида ИК изображений для обнаружения скрытых объектов Ч. 1. Решение на основе коэффициентной обратной задачи теплопроводности. Измерительная техника, 2013, 10, 47-50 [Ischuk I.N., Parfir'ev A.V. Reconstruction of the cuboid infrared imaging to detect concealed objects Part 1. The decision on the basis of the inverse heat conduction problem. Measurement technology, 2013, 10, 47-50 (in Russian)]

[6] Chulkov A.O., Vavilov V.P. Hardware and software for thermal nondestructive testing of metallic and composite materials. Journal of Physics: Conference Series, 2016, 671, 1-8.

[7] Demidova L.A., Sokolova Yu.S., Nikulchev E.V. Use of Fuzzy Clustering Algorithms Ensemble for SVM Classifier Development. International Review on Modelling and Simulations, 2015, 8(4), 446-457.

[8] Glenn Healey, David Slater. Models and Methods for Automated Material Identification in Hyperspectral Imagery Acquired Under Unknown Illumination and Atmospheric Conditions. Transactions On Geoscience And Remote Sensing, 1999, 37(6), 2706-2717. 